Proceedings of the Edinburgh Mathematical Society (2008) 51, 443-463 (C)

DOI:10.1017/S001309150500012X Printed in the United Kingdom

\title{
BOUNDEDNESS OF FOURIER INTEGRAL OPERATORS ON HARDY SPACES
}

\author{
MARCO M. PELOSO* AND SILVIA SECCO \\ Dipartimento di Matematica, Politecnico di Torino, 10129 Torino, Italy \\ (marco.peloso@polito.it; secco@calvino.polito.it)
}

(Received 1 February 2005)

Abstract For $0<p \leqslant 1$, let $h^{p}\left(\mathbb{R}^{n}\right)$ denote the local Hardy space. Let $\mathcal{F}$ be a Fourier integral operator defined by the oscillatory integral

$$
\mathcal{F} f(x)=\iint_{\mathbb{R}^{2 n}} \exp (2 \pi \mathrm{i}(\phi(x, \xi)-y \cdot \xi)) b(x, y, \xi) f(y) \mathrm{d} y \mathrm{~d} \xi,
$$

where $\phi$ is a $\mathcal{C}^{\infty}$ non-degenerate real phase function, and $b$ is a symbol of order $\mu$ and type $(\rho, 1-\rho)$, $\frac{1}{2}<\rho \leqslant 1$, vanishing for $x$ outside a compact set of $\mathbb{R}^{n}$. We show that when $p \leqslant 1$ and $\mu \leqslant-(n-$ $1)(1 / p-1 / 2)$ then $\mathcal{F}$ initially defined on Schwartz functions in $h^{p}\left(\mathbb{R}^{n}\right)$ extends to a bounded operator $\mathcal{F}: h^{p}\left(\mathbb{R}^{n}\right) \rightarrow h^{p}\left(\mathbb{R}^{n}\right)$. The range of $p$ and $\mu$ is sharp. This result extends to the local Hardy spaces the seminal result of Seeger et al. for the $L^{p}$ spaces. As immediate applications we prove the boundedness of smooth Radon transforms on hypersurfaces with non-vanishing Gaussian curvature on the local Hardy spaces.

Finally, we prove a local version for the boundedness of Fourier integral operators on local Hardy spaces on smooth Riemannian manifolds of bounded geometry.

Keywords: Fourier integral operators; Hardy spaces; Riesz transforms

2000 Mathematics subject classification: Primary 35S30; 42B30

Secondary $47 \mathrm{G} 30$

\section{Introduction}

Let $\mathcal{F}$ be a properly supported Fourier integral operator of order 0 and of type $(\rho, 1-\rho)$ associated with a local canonical graph. We defer this and other basic definitions to the next section. It was proved by Hörmander and Eskin $[\mathbf{3}, \mathbf{7}]$ when $\rho>\frac{1}{2}$ and by Beals $[\mathbf{1}]$ and Greenleaf and Uhlmann [5] when $\rho=\frac{1}{2}$ that the operator $\mathcal{F}$ is bounded on $L_{\text {loc }}^{2}$ and consequently a Fourier integral operator of order $\mu$ maps $L_{s \text {, comp }}^{2}$ into $L_{s-\mu \text {,loc }}^{2}$. Here and in what follows $L_{s}^{p}$ denotes the $L^{p}$-Sobolev space of order $s, L_{\text {loc }}^{p}$ denotes the space of functions $f$ such that $\psi f \in L^{p}$ for all compactly supported smooth functions $\psi$, and $L_{\text {comp }}^{p}=:\left(L_{\mathrm{loc}}^{p^{\prime}}\right)^{*}=L_{\mathrm{loc}}^{p} \cap \mathcal{E}^{\prime}$. For $p \neq 2$ it has been known for some time that a Fourier integral operator of order $\mu$ cannot be bounded on $L^{p}$ unless $\mu \leqslant-(n-1)|1 / p-1 / 2|,[\mathbf{1 1}]$.

* Present address: Dipartimento di Matematica, Università degli Studi di Milano, 20133 Milano, Italy (marco.peloso@mat.unimi.it). 
In [14] Seeger et al. proved that in fact this is the correct range for the $L^{p}$ boundedness for such an operator. They showed that if $\mathcal{F}$ is a Fourier integral operator of order $\mu$, then

$$
\mathcal{F}: L_{\text {comp }}^{p}\left(\mathbb{R}^{n}\right) \rightarrow L_{\text {loc }}^{p}\left(\mathbb{R}^{n}\right)
$$

boundedly, for $1<p<\infty$ and $\mu \leqslant-(n-1)|1 / p-1 / 2|$. To prove their theorem, Seeger et al. showed that, given two smooth cut-off functions $\psi_{1}, \psi_{2}$, the operator $\tilde{\mathcal{F}}=\psi_{2} \mathcal{F} \psi_{1}$ in fact maps $\tilde{\mathcal{F}}: h^{1}\left(\mathbb{R}^{n}\right) \rightarrow h^{1}\left(\mathbb{R}^{n}\right)$ continuously when $\mathcal{F}$ has order $-(n-1) / 2$. They then used interpolation for an analytic family of operators together with the $L^{2}$-result to obtain the result for the case when $1<p<2$. The result for $p>2$ then follows by duality, since the adjoint of $\mathcal{F}$ is again a Fourier integral operator of the same order.

In this paper we study the mapping properties of the operators $\mathcal{F}$ when acting on the local Hardy spaces $h^{p}$ in $\mathbb{R}^{n}$, introduced by Goldberg [4]. Our main result shows that, if $\mathcal{F}$ is a Fourier integral operator associated with a canonical graph, of order $\mu$, and if $\tilde{\mathcal{F}}$ is defined as above, then

$$
\tilde{\mathcal{F}}: h^{p}\left(\mathbb{R}^{n}\right) \rightarrow h^{p}\left(\mathbb{R}^{n}\right)
$$

is bounded, when $0<p \leqslant 1$ and $\mu \leqslant-(n-1)(1 / p-1 / 2)$, thus extending the Seeger et al. result to the local Hardy spaces $h^{p}$, with $0<p<1$. Finally, we extend this result to Riemannian manifolds of bounded geometry. For some previous related work, see [12].

The paper is organized as follows. We begin by giving the basic definitions. We also make a standard reduction that allows us to work with an operator defined by an oscillatory integral $T_{b}$ in the more familiar setting of $\mathbb{R}^{n}$. Here $b$ denotes the symbol in the class $S_{\rho, 1-\rho}^{\mu}$ (see (2.4), below). We first prove that such an operator $T_{b}$ is bounded from $h^{p}$ to $L^{p}$ (Theorem 2.2$)$, when $\mu \leqslant-(n-1)(1 / p-1 / 2)$ and $0<p \leqslant 1$. Section 3 is devoted to this. In $\S 4$ we use a characterization of local Hardy spaces in terms of local Riesz transforms (Proposition 4.1) to reduce the question of the boundedness of $T_{b}$ on $h^{p}$ to the uniform boundedness of operators of the form $P_{\varepsilon} T_{b}$ from $h^{p}$ to $L^{p}$, where $P_{\varepsilon}$ are suitable pseudodifferential operators of order 0 and type $(1,0)$. We remark that while Theorem 2.2 also holds when $\rho=\frac{1}{2}$, Proposition 4.2 fails to hold in this case. This is the reason for us to restrict ourselves to the case $\rho>\frac{1}{2}$.

In the last section we prove the extension of our main result, Theorem 2.1, to the case of a Fourier integral operator of order $\mu$ acting between paracompact smooth manifolds $X$ and $Y$ of bounded geometry (see Theorem 5.1).

\section{Basic definitions and statement of the main result}

Let $X$ and $Y$ be smooth paracompact manifolds of the same dimension $n$. Denote by $d \sigma_{X}$ and $d \sigma_{Y}$ the standard symplectic forms on $T^{*} X$ and $T^{*} Y$, respectively.

Let $\mathcal{C}$ be a conic Lagrangian submanifold of $T^{*} X \backslash\{0\} \times T^{*} Y \backslash\{0\}$, the latter being endowed with the symplectic form $d \sigma_{X}-d \sigma_{Y}$. We assume that $\mathcal{C}$ is locally the graph of a homogenous canonical transformation $H$. A smooth map $H$ from $T^{*} Y \backslash\{0\}$ to $T^{*} X \backslash\{0\}$ preserving the symplectic forms, i.e. such that $H^{*} d \sigma_{X}=d \sigma_{Y}$, is called a canonical transformation. Such a map $H$ turns out to always be a diffeomorphism. It is said to be homogeneous if $H(y, \lambda \eta)=\lambda H(y, \eta)$ for all $\lambda>0$ and $(y, \xi) \in T^{*} Y \backslash\{0\}$. 
We are concerned with operators $\mathcal{F} \in I^{\mu}(X \times Y, \mathcal{C})$, i.e. linear operators between the distribution spaces on $X$ and $Y$ whose kernels are Lagrangian distributions of order $\mu$ having their wavefront sets contained in

$$
\mathcal{C}^{\prime}=\{(x, \xi, y, \eta):(x, \xi, y,-\eta) \in \mathcal{C}\}
$$

We refer the reader to $[\mathbf{2}, \mathbf{6}, \mathbf{8}, \mathbf{1 8}]$ for the global theory of Fourier integral operators.

What is relevant to the present work is the local representation of Fourier integral operators. In local coordinates, such an operator $\mathcal{F}$ can be written as

$$
\mathcal{F} f(x)=\int_{\mathbb{R}^{n}} \int_{\mathbb{R}^{N}} \mathrm{e}^{2 \pi \mathrm{i} \Phi(x, y, \theta)} b(x, y, \theta) \mathrm{d} \theta f(y) \mathrm{d} y .
$$

Here $b$ denotes a classical symbol of order $\mu^{\prime}$, i.e. such that

$$
\left|\partial_{x, y}^{\beta} \partial_{\theta}^{\alpha} b(x, y, \theta)\right| \leqslant C_{\beta, \alpha}(1+|\theta|)^{\mu^{\prime}-|\alpha|}
$$

and the order $\mu^{\prime}=\mu-(N-n) / 2$. Moreover, the phase function $\Phi$ is real, positively homogenous of degree 1 in $\theta, C^{\infty}\left(\mathbb{R}^{2 n} \times \mathbb{R}^{N} \backslash\{0\}\right)$ and satisfies the following nondegeneracy conditions:

(i) $\mathrm{d} \Phi \neq 0$ everywhere (on the support of $b$ );

(ii) on the set $\Sigma_{\Phi}=\left\{\Phi_{\theta}=0\right\}$ the differentials $\mathrm{d} \Phi_{\theta_{1}}, \ldots, \mathrm{d} \Phi_{\theta_{N}}$ are linearly independent.

Henceforth, we denote by $\Phi_{\theta}, \Phi_{\theta_{j}}, \Phi_{x_{k}, \theta_{\ell}}$, etc., the partial derivatives $\partial_{\theta} \Phi, \partial_{\theta_{j}} \Phi, \partial_{x_{j} \theta_{\ell}}^{2} \Phi$, etc., of $\Phi$ with respect to the indicated variables.

By Hörmander's theorem on the equivalence of phase functions (see [18, Proposition VIII 1.5]) one can reduce the number $N$ of frequency variables to $n$, so that, after this reduction, the order of the Fourier integral operator $\mathcal{F}$ coincides with the order of the symbol $b$.

Possibly by multiplying by a smooth cut-off function, one may assume that, modulo a smoothing operator, the symbol $b$ is supported in a small conic neighbourhood of $\Sigma_{\Phi}$. Then, it turns out that the mapping

$$
(x, y, \theta) \mapsto\left(x, \Phi_{x}(x, y, \theta), y,-\Phi_{y}(x, y, \theta)\right)
$$

is a smooth mapping onto a conic open set of $T^{*} X \backslash\{0\} \times T^{*} Y \backslash\{0\}$, sending $\Sigma_{\Phi}$ onto a conic Lagrangian submanifold $\mathcal{C}^{\prime}$ such that the wavefront set of $\mathcal{F}$ satisfies

$$
W F(\mathcal{F}) \subseteq \mathcal{C}^{\prime}=\left\{\left(x, \Phi_{x}(x, y, \theta), y, \Phi_{y}(x, y, \theta)\right): \Phi_{\theta}(x, y, \theta)=0\right\}
$$

The local Hardy spaces on $\mathbb{R}^{n}$ were introduced by Goldberg [4] (see also [17, Chapter III.5]. They can be described as follows. Let $\Psi$ be a fixed Schwartz function, $\int \Psi=1$. For a tempered distribution $f$ let $\mathcal{M}_{\Psi}$ be the maximal operator

$$
\mathcal{M}_{\Psi} f(x)=\sup _{0<t \leqslant 1}\left|f * \Psi_{t}(x)\right|,
$$


where, as usual, $\Psi_{t}(x)=t^{-n} \Psi(x / t)$. Let $0<p \leqslant 1$. We say that a tempered distribution $f$ is in $h^{p}\left(\mathbb{R}^{n}\right)$ if

$$
\|f\|_{h^{p}\left(\mathbb{R}^{n}\right)}:=\left\|\mathcal{M}_{\Psi}(f)\right\|_{L^{p}\left(\mathbb{R}^{n}\right)}<\infty .
$$

We are now in a position to state our main result.

Theorem 2.1. Let $\mathcal{C} \subseteq T^{*}\left(\mathbb{R}^{n}\right) \backslash\{0\} \times T^{*}\left(\mathbb{R}^{n}\right) \backslash\{0\}$ be a submanifold which is locally a canonical graph and let $\mathcal{F} \in I^{\mu}\left(\mathbb{R}^{n} \times \mathbb{R}^{n}, \mathcal{C}\right)$. Let $h^{p}\left(\mathbb{R}^{n}\right)$ denote the local Hardy space for $0<p \leqslant 1$ and let $\mu \leqslant-(n-1)(1 / p-1 / 2)$. For $\psi_{1}, \psi_{2}$ smooth cut-off functions define $\tilde{\mathcal{F}}=\psi_{1} \mathcal{F} \psi_{2}$. Then

$$
\tilde{\mathcal{F}}: h^{p}\left(\mathbb{R}^{n}\right) \rightarrow h^{p}\left(\mathbb{R}^{n}\right)
$$

is bounded.

We can extend this result to a class of paracompact smooth manifolds (see Theorem 5.1).

Moreover, we can allow the symbol $b$ to belong to the more general class $S_{\rho, 1-\rho}^{\mu}$, with $\frac{1}{2}<\rho \leqslant 1$ (see Theorem 2.2).

Now we make a reduction that allows us to study the boundedness of an operator defined by an oscillatory integral in $\mathbb{R}^{2 n}$.

The assumption that the conic Lagrangian manifold $\mathcal{C}$ is locally the graph of such a map $H$, i.e. that $\mathcal{C}$ has the form $\mathcal{C}=\left\{(H(y, \xi),(y, \xi)):(y, \xi) \in T^{*} Y \backslash\{0\}\right\}$, allows us to make the following reduction. Given any point $\left(x_{0}, \xi_{0}, y_{0}, \eta_{0}\right) \in \mathcal{C}$, there exists a neighbourhood on which one can choose local coordinates so that

$$
\mathcal{C}=\left\{\left(x, \phi_{x}, \phi_{\eta}, \eta\right)\right\}
$$

for some smooth phase function $\phi=\phi(x, \eta)$ such that

$$
\phi_{\eta}\left(x_{0}, \eta_{0}\right)=y_{0} .
$$

Note that in this case, the phase function $\Phi(x, y, \xi)$ in these coordinates has the expression $\Phi(x, y, \theta)=\phi(x, \theta)-y \cdot \theta$ and the operator $\mathcal{F}$ takes the form

$$
\mathcal{F} f(x)=\iint_{\mathbb{R}^{2 n}} \exp (2 \pi \mathrm{i}(\phi(x, \xi)-y \cdot \xi)) b(x, y, \xi) f(y) \mathrm{d} \xi \mathrm{d} y .
$$

(In order to use more familiar notation, we henceforth rename the variable $\theta$ as $\xi$.)

Since $\mathcal{C}$ is locally the graph of a diffeomorphism, the $\phi(x, \xi)$ function turns out to be real-valued, $\mathcal{C}^{\infty}\left(\mathbb{R}^{n} \backslash\{0\}\right)$ and homogeneous of degree 1 in $\xi$, and moreover

$$
\operatorname{det}\left(\phi_{x_{i} \xi_{j}}\right) \neq 0
$$

for $\xi \neq 0$ and $(x, \xi)$ in the support of $b$.

As mentioned above, we can allow the symbol $b$ to vary in a more general class, which now we define. Let $\frac{1}{2}<\rho \leqslant 1$. One says that $b \in S_{(\rho, 1-\rho)}^{\mu}$ if $b \in \mathcal{C}^{\infty}\left(\mathbb{R}^{2 n} \times \mathbb{R}^{n}\right)$ satisfies the estimates

$$
\left|\partial_{x, y}^{\beta} \partial_{\xi}^{\alpha} b(x, y, \xi)\right| \leqslant C_{\alpha, \beta}(1+|\xi|)^{\mu-\rho|\alpha|+(1-\rho)|\beta|}
$$


for some constants $C_{\alpha, \beta}$ and all multi-indices $\alpha, \beta$ and $x, \xi \in \mathbb{R}^{n}$. We also assume that $b$ vanishes for $x$ outside a fixed compact set.

Consider the Fourier integral operator $T=T_{b}$ with symbol $b$ and phase $\phi$

$$
T(f)(x)=\iint_{\mathbb{R}^{2 n}} \exp (2 \pi \mathrm{i}(\phi(x, \xi)-y \cdot \xi)) b(x, y, \xi) f(y) \mathrm{d} y \mathrm{~d} \xi,
$$

initially defined for $f$ in the Schwartz class $\mathcal{S}\left(\mathbb{R}^{n}\right)$.

For this kind of operator, our main result is the following.

Theorem 2.2. Let $T$ be defined as in (2.5) with symbol $b \in S_{(\rho, 1-\rho)}^{\mu}$. Let $0<p \leqslant 1$ and suppose that

$$
\mu \leqslant-(n-\rho)\left(\frac{1}{p}-\frac{1}{2}\right)
$$

Then $T$, initially defined on Schwartz functions, extends to a bounded linear operator

$$
T: h^{p}\left(\mathbb{R}^{n}\right) \rightarrow h^{p}\left(\mathbb{R}^{n}\right) .
$$

Note that, in this setting, we need not require the symbol $b$ to be also compactly supported in the $y$-variable.

In order to state our next main result, we need a few more definitions. Suppose that, with any $x$ varying in an open set $\Omega$ in $\mathbb{R}^{n}$ is associated a hypersurface $H_{x}$ with non-zero Gaussian curvature and surface measure $\mathrm{d} s_{x}$. We assume that the mapping $x \mapsto H_{x}$ is smooth in $\Omega$. Let $\psi \in \mathcal{C}_{0}^{\infty}\left(\Omega \times \mathbb{R}^{n}\right)$ and let $\mathrm{d} \lambda(x, y)=\psi(x, y) \mathrm{d} s_{x}(y)$.

We will denote the Fourier transform of a tempered distribution $f$ by $\hat{f}$. If $f$ is integrable, we have

$$
\hat{f}(\xi)=\int_{\mathbb{R}^{n}} \exp (-2 \pi \mathrm{i} \xi \cdot x) f(x) \mathrm{d} x .
$$

For $f \in \mathcal{S}\left(\mathbb{R}^{n}\right)$ we define the smooth Radon transform as the operator

$$
R(f)(x)=(\mathrm{d} \lambda(x, \cdot) * f)(x),
$$

i.e.

$$
R(f)(x)=\int \exp (2 \pi \mathrm{i} x \cdot \xi) \widehat{\mathrm{d}(x, \cdot)}(\xi) \hat{f}(\xi) \mathrm{d} \xi .
$$

For $0<p \leqslant 1$ and $s \in \mathbb{R}^{n}$ we define the potential local Hardy spaces

$$
h_{s}^{p}\left(\mathbb{R}^{n}\right)=\left\{f \in \mathcal{S}^{\prime}: f, \Lambda_{s} f \in h^{p}\left(\mathbb{R}^{n}\right)\right\},
$$

where $\widehat{\left(\Lambda_{s} f\right)}(\xi)=\left(1+|\xi|^{2}\right)^{s / 2} \hat{f}(\xi)$.

Theorem 2.3. Let $R$ be a smooth Radon transform as in (2.6). Then, initially defined on Schwartz functions, $R$ extends to a bounded linear operator

$$
R: h_{s}^{p}\left(\mathbb{R}^{n}\right) \rightarrow h^{p}\left(\mathbb{R}^{n}\right)
$$

if $s \geqslant(n-1)(1 / p-1)$. 
Consider an operator of the form (2.5). If we assume further that $b$ also has compact support in the $\xi$-variable, then $T f$ can be written as an integral operator with kernel

$$
K(x, y)=\int_{\mathbb{R}^{n}} \exp (2 \pi \mathrm{i}(\phi(x, \xi)-y \cdot \xi)) b(x, y, \xi) \mathrm{d} \xi,
$$

which is singular at the points $(x, y)$ such that

$$
\nabla_{\xi}(\phi(x, \xi)-y \cdot \xi)=0 .
$$

Henceforth, we write $\phi_{\xi}$ in place of $\nabla_{\xi} \phi$. Then, for $x \in \mathbb{R}^{n}$, the set of singular points for the kernel $K$ is

$$
\Sigma_{x}=\left\{y \in \mathbb{R}^{n}: y=\phi_{\xi}(x, \xi) \text { for some } \xi \in \mathbb{R}^{n}\right\} .
$$

We now have our main $h^{p}$ to $L^{p}$ result.

Theorem 2.4. Let $T$ be a Fourier integral operator defined by the oscillatory integral as in (2.5), where $b \in S_{(\rho, 1-\rho)}^{\mu}$. Suppose that

$$
\mu<-\frac{n-\rho}{2} \text { and } \quad \frac{1}{p}-\frac{1}{2}=-\frac{\mu}{n-\rho} .
$$

Then $T$, initially defined on the dense subspace $\mathcal{S}\left(\mathbb{R}^{n}\right)$, extends to a bounded linear operator

$$
T: h^{p}\left(\mathbb{R}^{n}\right) \rightarrow L^{p}\left(\mathbb{R}^{n}\right) .
$$

\section{Proof of Theorem 2.4}

A distribution $f \in h^{p}\left(\mathbb{R}^{n}\right)$ admits an atomic decomposition

$$
f=\sum_{j} \lambda_{j} a_{j}
$$

where the $\lambda_{j}$ are constants such that $\sum_{j}\left|\lambda_{j}\right|^{p} \approx\|f\|_{h^{p}\left(\mathbb{R}^{n}\right)}^{p}$ and the $a_{j}$ are $p$-atoms. A function $a$ is called a $p$-atom if

(i) $\operatorname{supp} a \subseteq B\left(x_{0}, \delta\right)$,

(ii) $|a(x)| \leqslant|B|^{-1 / p}$,

(iii) $\int x^{\alpha} a(x) \mathrm{d} x=0$ if $\delta \leqslant 1$ and $|\alpha| \leqslant M=1+[n(1 / p-1)]$, and no further condition is required if $\delta>1$.

Here $[x]$ denotes the integral part of the real number $x$ and we denote by $B\left(x_{0}, \delta\right)$ the ball centred at $x_{0}$ and of radius $\delta$.

We recall that the Schwartz space $\mathcal{S}\left(\mathbb{R}^{n}\right)$ is contained and dense in $h^{p}\left(\mathbb{R}^{n}\right)($ see $[\mathbf{4}, \mathbf{1 3}])$.

Using the atomic decomposition of $h^{p}\left(\mathbb{R}^{n}\right)$, it suffices to show that there exists a constant $A>0$ such that, for all $p$-atoms $a$,

$$
\int|T a(x)|^{p} \mathrm{~d} x \leqslant A .
$$


We first assume that the atom $a$ has support in a ball $B$ of radius $\delta>1$. Note that since $\mu<0, T$ is bounded from $L^{2}$ into itself. Let $r=2 / p$. By applying Hölder's inequality with $r$ and its conjugate exponent $r^{\prime}$ we see that

$$
\begin{aligned}
\int|T a(x)|^{p} \mathrm{~d} x & \leqslant|\operatorname{supp}(b)|^{1-p / 2}\left(\int|T a(x)|^{2} \mathrm{~d} x\right)^{p / 2} \\
& \leqslant|\operatorname{supp}(b)|^{1-p / 2}\|T\|_{\left(L^{2}, L^{2}\right)}\|a\|_{2}^{p} \\
& =C\|a\|_{2}^{p} .
\end{aligned}
$$

Next,

$$
\begin{aligned}
\|a\|_{2}^{p} & =\left(\int_{B}|a(x)|^{2} \mathrm{~d} x\right)^{p / 2} \leqslant\left(\int_{B}|B|^{-2 / p} \mathrm{~d} x\right)^{p / 2} \\
& \leqslant|B|^{-1+p / 2}=c_{n} \delta^{n(-1+p / 2)} .
\end{aligned}
$$

Since $\delta>1$, we obtain that

$$
\int|T a(x)|^{p} \mathrm{~d} x \leqslant A,
$$

with $A$ independent of the atom $a$ and $\delta>1$.

Next we consider the case of an atom $a$ supported on a ball $B$ of radius $\delta \leqslant 1$. For $x \in \mathbb{R}^{n}$, let $\Sigma_{x}$ be the singular region defined in (2.8). Recall that the set $\{(x, y)$ : $\left.y \in \Sigma_{x}\right\}$ contains the singularity of the kernel $K$ of the operator $T$. We will now adapt the decomposition of the cotangent space introduced by Seeger et al. [14] to the present situation.

For each integer $j \geqslant 1$ we fix a collection of unit vectors $\left\{\xi_{j}^{\nu}\right\}$ such that

(i) $\left|\xi_{j}^{\nu}-\xi_{j}^{\nu^{\prime}}\right| \geqslant 2^{-j / 2}$ if $\nu \neq \nu^{\prime}$,

(ii) if $\xi$ is any unit vector, then there exists a unit vector $\xi_{j}^{\nu}$ such that $\left|\xi-\xi_{j}^{\nu}\right| \leqslant 2^{-j / 2}$.

Recall that it suffices to take a collection $\left\{\xi_{j}^{\nu}\right\}$ which is maximal with respect to (i). It should also be noted that there are at most $c 2^{j(n-1) / 2}$ elements in the collection $\left\{\xi_{j}^{\nu}\right\}$.

Consider now the ball $B=B(\bar{y}, \delta)$ of centre $\bar{y}$ and radius $\delta \leqslant 1$. We first define the sets $\tilde{R}_{j}^{\nu}$ as

$$
\tilde{R}_{j}^{\nu}=\left\{y \in \mathbb{R}^{n}:|y-\bar{y}| \leqslant \bar{c} 2^{-j / 2},\left|\pi_{j}^{\nu}(y-\bar{y})\right| \leqslant \bar{c} 2^{-j \rho}\right\},
$$

where $\pi_{j}^{\nu}$ denotes the orthogonal projection in the direction of $\xi_{j}^{\nu}$ and $\bar{c}$ is a constant independent of $j$ to be fixed later. Note that

$$
\left|\tilde{R}_{j}^{\nu}\right| \approx 2^{-(n-1) j / 2} \cdot 2^{-j \rho}=2^{-(n-1+2 \rho) j / 2}
$$

By (2.3), for every $\xi$, the mapping

$$
x \mapsto y=\phi_{\xi}(x, \xi)=\left(\partial_{\xi_{1}} \phi(x, \xi), \ldots, \partial_{\xi_{n}} \phi(x, \xi)\right)
$$


has a nowhere-vanishing Jacobian, i.e.

$$
\left|\operatorname{det} j a c\left(x \mapsto y=\phi_{\xi}(x, \xi)\right)\right|=\left|\operatorname{det}\left(\phi_{x_{j} \xi_{k}}\right)(x, \xi)\right| \geqslant \kappa>0,
$$

with $\kappa$ independent of $x$ and $\xi$ on the unit sphere.

We define $R_{j}^{\nu}$ to be the preimage of $\tilde{R}_{j}^{\nu}$ under the mapping $\phi_{\xi}$ with $\xi=\xi_{j}^{\nu}$ :

$$
R_{j}^{\nu}=\left\{x \in \mathbb{R}^{n}:\left|\bar{y}-\phi_{\xi}\left(x, \xi_{j}^{\nu}\right)\right| \leqslant \bar{c} 2^{-j / 2},\left|\pi_{j}^{\nu}\left(\bar{y}-\phi_{\xi}\left(x, \xi_{j}^{\nu}\right)\right)\right| \leqslant \bar{c} 2^{-j \rho}\right\} .
$$

Now

$$
\left|\tilde{R}_{j}^{\nu}\right|=\int_{R_{j}^{\nu}}\left|\operatorname{det}\left(\phi_{x_{j} \xi_{k}}\right)(x, \xi)\right| \mathrm{d} x \geqslant \kappa \int_{R_{j}^{\nu}} \mathrm{d} x=\kappa\left|R_{j}^{\nu}\right|
$$

so that

$$
\left|R_{j}^{\nu}\right| \leqslant \kappa^{-1}\left|\tilde{R}_{j}^{\nu}\right| .
$$

Thus, given the ball $B=B(\bar{y}, \delta)$ we define

$$
B^{*}=\bigcup_{2^{-j} \leqslant \delta} \bigcup_{\nu} R_{j}^{\nu}
$$

Note that, by the above estimate for $\left|R_{j}^{\nu}\right|$ and (3.2), we obtain that

$$
\left|B^{*}\right| \leqslant \sum_{2^{-j} \leqslant \delta} \sum_{\nu}\left|R_{j}^{\nu}\right| \leqslant c \sum_{2^{-j} \leqslant \delta} 2^{-(n-1+2 \rho) j / 2} 2^{(n-1) j / 2} \leqslant c \delta^{\rho} .
$$

We wish to estimate

$$
\int_{\mathbb{R}^{n}}|T a(x)|^{p} \mathrm{~d} x=\int_{B^{*}}|T a(x)|^{p} \mathrm{~d} x+\int_{c^{*}}|T a(x)|^{p} \mathrm{~d} x,
$$

where $B$ is the ball of radius $\delta \leqslant 1$ containing the support of the atom $a$ and $B^{*}$ is defined in (3.4).

By Hölder's inequality, we have

$$
\begin{aligned}
\int_{B^{*}}|T a(x)|^{p} \mathrm{~d} x & \leqslant\left|B^{*}\right|^{1-p / 2}\left(\int_{B^{*}}|T a(x)|^{2} \mathrm{~d} x\right)^{p / 2} \\
& \leqslant c \delta^{(1-p / 2) \rho}\|T a\|_{L^{2}}^{p} .
\end{aligned}
$$

We distinguish two cases. Suppose first that

$$
-\frac{n}{2}<\mu<-\frac{n-\rho}{2} .
$$

The operator $T$ is then bounded from $L^{q}\left(\mathbb{R}^{n}\right)$ to $L^{2}\left(\mathbb{R}^{n}\right)$ when

$$
\frac{1}{2}=\frac{1}{q}-\frac{\mu}{n}
$$


(see [17, Chapter IX.3]). Hence, in this case,

$$
\|T a\|_{L^{2}}^{p} \leqslant\|T\|_{\left(L^{q}, L^{2}\right)}\|a\|_{L^{q}}^{p} \leqslant c|B|^{-1+p / q} \leqslant c \delta^{n(-1+p / q)} .
$$

Therefore, we obtain that

$$
\int_{B^{*}}|T a(x)|^{p} \mathrm{~d} x \leqslant c \delta^{(1-p / 2) \rho+n(-1+p / q)} \leqslant c,
$$

using the above condition, (3.7) and the relation between $p$ and $\rho$.

We now assume that

$$
\mu \leqslant-\frac{1}{2} n
$$

If we set

$$
\tilde{a}=|B|^{1 / p-1 / q} a
$$

then $\tilde{a}$ becomes a $q$-atom, with support contained in the same ball $B$ as $a$. Therefore, $\tilde{a}$ is also an element of $H^{q}\left(\mathbb{R}^{n}\right)$ and in fact an atom of $H^{q}\left(\mathbb{R}^{n}\right)$. Since $T: H^{q}\left(\mathbb{R}^{n}\right) \rightarrow L^{2}\left(\mathbb{R}^{n}\right)$ is bounded when $1 / 2=1 / q-\mu / n[\mathbf{1 0}]$ (see also [17, Chapter III]) we have

$$
\begin{aligned}
\int_{B^{*}}|T a(x)|^{p} \mathrm{~d} x & \leqslant c \delta^{(1-p / 2) \rho}\|T\|_{\left(H^{q}, L^{2}\right)}\|a\|_{H^{q}}^{p} \\
& \leqslant c \delta^{(1-p / 2) \rho}|B|^{(1 / q-1 / p) p}\|\tilde{a}\|_{H^{q}}^{p} \\
& \leqslant c \delta^{(1-p / 2) \rho+n(p / q-1)} \\
& \leqslant c
\end{aligned}
$$

by the same argument as before.

We now turn to the estimate of the second integral on the right-hand side of (3.6). Also in this case, we will adapt the decomposition of the kernel $K$ of the operator $T$ into kernels $K_{j}^{\nu}$ constructed by Seeger et al. in [14].

Recall that we have defined $M=[n(1 / p-1)]+1$. Now we set

$$
\gamma=\frac{M(1+n(1 / p-1))}{(M-n(1 / p-1)) \rho}
$$

Note that $\gamma \geqslant 1 / \rho \geqslant 1$.

Consider the collection of shells $\left\{\mathcal{C}_{j}\right\}, j=0,1,2, \ldots$, where

$$
\mathcal{C}_{0}=\left\{\xi \in \mathbb{R}^{n}:|\xi| \leqslant 2^{1 / \gamma}\right\}
$$

and

$$
\mathcal{C}_{j}=\left\{\xi \in \mathbb{R}^{n}: 2^{(j-1) / \gamma} \leqslant|\xi| \leqslant 2^{(j+1) / \gamma}\right\}, \quad j=1,2, \ldots
$$

For $j>0$ fixed, we consider the collection of unit vectors $\left\{\xi_{j}^{\nu}\right\}_{\nu}$ as above. Set

$$
\Gamma_{j}^{\nu}=\left\{\xi \in \mathbb{R}^{n}:\left|\xi_{j}^{\nu}-\frac{\xi}{|\xi|}\right| \leqslant 2 \cdot 2^{-j / 2}\right\} .
$$


We now construct a partition of unity associated with the $\Gamma_{j}^{\nu}$. Let $\tilde{\phi}$ be a non-negative function in $\mathcal{C}_{0}^{\infty}\left(\mathbb{R}^{n}\right)$ such that $\tilde{\phi}(y)=1$ when $|y| \leqslant 1$ and $\tilde{\phi}(y)=0$ when $|y| \geqslant 2$. Set

$$
\eta_{j}^{\nu}(\xi)=\tilde{\phi}\left(2^{j / 2}\left(\frac{\xi}{|\xi|}-\xi_{j}^{\nu}\right)\right), \quad \xi \neq 0 .
$$

Then $\eta_{j}^{\nu} \in \mathcal{C}_{0}^{\infty}\left(\mathbb{R}^{n} \backslash\{0\}\right)$ is homogeneous of degree 0 in $\xi$, with support on the cone $\Gamma_{j}^{\nu}$ and identically 1 on the cone

$$
\left\{\xi \in \mathbb{R}^{n}:\left|\xi_{j}^{\nu}-\frac{\xi}{|\xi|}\right| \leqslant 2^{-j / 2}\right\}
$$

Since $\sum_{\nu} \eta_{j}^{\nu}(\xi) \neq 0$ for all $\xi \neq 0$, we set

$$
\chi_{j}^{\nu}(\xi)=\eta_{j}^{\nu}(\xi)\left(\sum_{\nu} \eta_{j}^{\nu}(\xi)\right)^{-1},
$$

so that, for every $j=1,2, \ldots$,

$$
\sum_{\nu} \chi_{j}^{\nu}(\xi)=1 \quad \text { for all } \xi \neq 0
$$

Let $\theta$ be $\mathcal{C}^{\infty}$ with support on the interval $\left(2^{-1 / \gamma}, 2^{1 / \gamma}\right)$ and let it satisfy

$$
\sum_{k \in \mathbb{Z}} \theta\left(2^{-k / \gamma} t\right)=1 \quad \text { for all } t>0
$$

For $j>0$ set

$$
\hat{\psi}_{j}(\xi)=\theta\left(2^{-j / \gamma}|\xi|\right) \quad \text { and } \quad \hat{\psi}_{0}(\xi)=1-\sum_{j>0} \hat{\psi}_{j}(\xi) .
$$

It then follows that, for all $\xi \in \mathbb{R}^{n}$,

$$
1=\hat{\psi}_{0}(\xi)+\sum_{j=1}^{+\infty} \sum_{\nu} \chi_{j}^{\nu}(\xi) \hat{\psi}_{j}(\xi) .
$$

Using this decomposition we then write

$$
T=\sum_{j=0}^{+\infty} T_{j}=\sum_{j} \sum_{\nu} T_{j}^{\nu},
$$

where

$$
\begin{aligned}
T_{j}^{\nu} f(x) & =\iint_{\mathbb{R}^{2 n}} b_{j}^{\nu}(x, y, \xi) \exp (2 \pi \mathrm{i}(\phi(x, \xi)-y \cdot \xi)) f(y) \mathrm{d} \xi \mathrm{d} y \\
& =\int_{\mathbb{R}^{n}} K_{j}^{\nu}(x, y) f(y) \mathrm{d} y, \\
K_{j}^{\nu}(x, y) & =\int_{\mathbb{R}^{n}} b_{j}^{\nu}(x, y, \xi) \exp (2 \pi \mathrm{i}(\phi(x, \xi)-y \cdot \xi)) \mathrm{d} \xi
\end{aligned}
$$


and

$$
\begin{aligned}
& b_{0}^{\nu}(x, y, \xi)=b_{0}(x, y, \xi)=b(x, y, \xi) \hat{\psi}_{0}(\xi), \\
& b_{j}^{\nu}(x, y, \xi)=b_{j}(x, y, \xi) \chi_{j}^{\nu}(x, \xi)=b(x, y, \xi) \hat{\psi}_{j}(\xi) \chi_{j}^{\nu}(\xi), \quad j=1,2, \ldots
\end{aligned}
$$

Note that for $j>0$ the symbol $b_{j}^{\nu}$ has support in the $\xi$-variables in the region $\mathcal{C}_{j} \cap \Gamma_{j}^{\nu}$ and that the surface measure of the set $\Gamma_{j}^{\nu}$ intersected with the unit sphere has a size approximately equal to $2^{-j / 2}$. Thus, the 'radius' of $\Gamma_{j}^{\nu}$ on the sphere of radius $2^{j / \gamma}$ is $2^{j(2-\gamma) / 2 \gamma}$.

We wish to prove some estimates for the kernels $K_{j}$ of the operators $T_{j}$. In order to do this, it suffices to prove appropriate estimates for the kernels $K_{j}^{\nu}$.

As in [14] we linearize the phase $\phi(x, \xi)-y \cdot \xi$ when $\xi$ is in $\operatorname{supp}\left(b_{j}^{\nu}\right)$. For simplicity, we write $\xi_{j}^{\nu}=\bar{\xi}$ and we select coordinates in the $\xi$-variables so that $\xi_{1}$ is the direction of $\bar{\xi}$ and $\xi^{\prime}=\left(\xi_{2}, \ldots, \xi_{n}\right)$ is orthogonal to $\bar{\xi}$.

We then write

$$
\phi(x, \xi)-y \cdot \xi=\left(\phi_{\xi}(x, \bar{\xi})-y\right) \cdot \xi-\left(\phi(x, \xi)-\phi_{\xi}(x, \bar{\xi}) \cdot \xi\right)
$$

and we set

$$
h_{x}(\xi)=\phi(x, \xi)-\phi_{\xi}(x, \bar{\xi}) \cdot \xi
$$

The two estimates in the following lemma can be proved by [14, (3.17) and (3.18)] (or see $[\mathbf{1 7}$, Chapter IX]).

Lemma 3.1. The function $h_{x}$ satisfies the estimates

(i) $\left|\left(\partial_{\xi_{1}}\right)^{N} h_{x}(\xi)\right| \leqslant A_{N} 2^{-N j / \gamma}$,

(ii) $\left|\left(\nabla_{\xi^{\prime}}\right)^{N} h_{x}(\xi)\right| \leqslant A_{N} 2^{-N j / 2 \gamma}$,

for any integer $N \geqslant 1$ and $(x, \xi)$ in the support of $b_{j}^{\nu}$.

We note that the condition $\xi$ in the support of $b_{j}^{\nu}$ in the present choice of coordinates translates into

$$
2^{(j-1) / \gamma} \leqslant|\xi| \leqslant 2^{(j+1) / \gamma} \text { and }\left|\xi^{\prime}\right| \leqslant c \cdot 2^{j(2-\gamma) / 2 \gamma} .
$$

\subsection{Estimates for the kernel $K_{j}$ sizes}

In order to complete the proof of Theorem 2.4 we need the following estimates for sizes of the kernel $K_{j}$.

Proposition 3.2. The kernels $K_{j}$ satisfy the following estimates:

(i) for all $y \in \mathbb{R}^{n}$,

$$
\int_{\mathbb{R}^{n}}\left|K_{j}(x, y)\right| \mathrm{d} x \leqslant A
$$


(ii) for all $y, y^{\prime} \in \mathbb{R}^{n}$,

$$
\int_{\mathbb{R}^{n}}\left|K_{j}(x, y)-q_{j}\left(x, y-y^{\prime}\right)\right| \mathrm{d} x \leqslant A 2^{j M / \gamma}\left|y-y^{\prime}\right|^{M},
$$

where $q_{j}\left(x, y-y^{\prime}\right)$ is the Taylor polynomial of $K_{j}$ centred at $y^{\prime}$ of order $M-1$;

(iii) for $y \in B$ and $2^{-j} \leqslant \delta$,

$$
\int_{c_{B^{*}}}\left|K_{j}(x, y)\right| \mathrm{d} x \leqslant A 2^{-j \rho} \delta^{-1} .
$$

The constant $A>0$ is independent of $j, y, y^{\prime}$ and $\delta$.

The argument is somewhat standard, but we include it for sake of completeness. We write the kernel $K_{j}^{\nu}$ by linearizing the phase and including the error made in this process into the symbol. Explicitly,

$$
\begin{aligned}
K_{j}^{\nu}(x, y) & =\int_{\mathbb{R}^{n}} \exp (2 \pi \mathrm{i}(\phi(x, \xi)-y \cdot \xi)) b_{j}^{\nu}(x, y, \xi) \mathrm{d} \xi \\
& =\int_{\mathbb{R}^{n}} \exp \left(2 \pi \mathrm{i}\left(\phi_{\xi}(x, \bar{\xi})-y\right) \cdot \xi\right) \tau_{j}^{\nu}(x, y, \xi) \mathrm{d} \xi
\end{aligned}
$$

where

$$
\tau_{j}^{\nu}(x, y, \xi)=b_{j}^{\nu}(x, y, \xi) \exp \left(2 \pi \mathrm{i} h_{x}(\xi)\right) .
$$

We consider the partial differential operator

$$
L=\left(I-2^{2 j} \partial_{\xi_{1}^{2}}^{2}\right)\left(I-2^{j \rho}\left\langle\nabla_{\xi^{\prime}}, \nabla_{\xi^{\prime}}\right\rangle\right) .
$$

It is easy to check that for every positive integer $N$

$$
\begin{aligned}
L^{N}\left(\exp \left(2 \pi \mathrm{i}\left(\phi_{\xi}(x, \bar{\xi})-y\right) \cdot \xi\right)\right)= & \exp \left(2 \pi \mathrm{i}\left(\phi_{\xi}(x, \bar{\xi})-y\right) \cdot \xi\right) \\
& \times\left(1+4 \pi^{2}\left|2^{j}\left(\phi_{\xi}(x, \bar{\xi})-y\right)_{1}\right|^{2}\right)^{N} \\
& \times\left(1+4 \pi^{2}\left|2^{j \rho / 2}\left(\phi_{\xi}(x, \bar{\xi})-y\right)^{\prime}\right|^{2}\right)^{N}
\end{aligned}
$$

where $v_{1}$ denotes the first component of the vector $v$, while $v^{\prime}$ denotes the vector $\left(v_{2}, \ldots, v_{n}\right)$.

Recalling that $\tau_{j}^{\nu}(x, y, \xi)=b_{j}^{\nu}(x, y, \xi) \exp \left(2 \pi \mathrm{i} h_{x}(\xi)\right)$ and the estimates for $b_{j}^{\nu}$ we easily see that for all non-negative integers $N$

$$
\left|L^{N} \tau_{j}^{\nu}(x, y, \xi)\right| \leqslant A_{N} 2^{-j(n-\rho) / 2 \gamma} .
$$

Then, by integration by parts we obtain

$$
\begin{array}{r}
\left|K_{j}^{\nu}(x, y)\right| \leqslant\left(1+4 \pi^{2}\left|2^{j}\left(\phi_{\xi}(x, \bar{\xi})-y\right)_{1}\right|^{2}\right)^{-N}\left(1+4 \pi^{2}\left|2^{j \rho / 2}\left(\phi_{\xi}(x, \bar{\xi})-y\right)^{\prime}\right|^{2}\right)^{-N} \\
\times \int_{\operatorname{supp}\left(\tau_{j}^{\nu}\right)}\left|L^{N} \tau_{j}^{\nu}(x, y, \xi)\right| \mathrm{d} \xi \\
\leqslant\left(1+4 \pi^{2}\left|2^{j}\left(\phi_{\xi}(x, \bar{\xi})-y\right)_{1}\right|^{2}\right)^{-N}\left(1+4 \pi^{2}\left|2^{j \rho / 2}\left(\phi_{\xi}(x, \bar{\xi})-y\right)^{\prime}\right|^{2}\right)^{-N} \\
\times A_{N} 2^{-j(n-\rho) / 2 \gamma} \cdot 2^{j / \gamma} \cdot 2^{(n-1) j(2-\gamma) / 2 \gamma} .
\end{array}
$$


Next we take $N>n / 2$ and integrate the above inequality in $x \in \mathbb{R}^{n}$.

We make the change of variables $z=\phi_{\xi}(x, \bar{\xi})$ and recall that the determinant Jacobian of this map is bounded below by the uniform positive constant $\kappa$ :

$$
\begin{aligned}
\int_{\mathbb{R}^{n}}\left|K_{j}^{\nu}(x, y)\right| \mathrm{d} x & \leqslant C_{N} \int_{\mathbb{R}^{n}} \frac{2^{j(2-(n-\rho)+(n-1)(2-\gamma)) / 2 \gamma}}{\left(1+4 \pi^{2}\left|2^{j}(z-y) 1\right|^{2}+4 \pi^{2}\left|2^{j \rho / 2}(z-y)^{\prime}\right|^{2}\right)^{N}} \mathrm{~d} z \\
& =C_{N} \int_{\mathbb{R}^{n}} \frac{2^{j(2-(n-\rho)+(n-1)(2-\gamma)-2 \gamma-(n-1) \rho \gamma) / 2 \gamma}}{\left(1+4 \pi^{2}|u|^{2}\right)^{N}} \mathrm{~d} u \\
& =C_{N} 2^{j(n+\rho-\gamma(n \rho+2-\rho))} \cdot 2^{-j(n-1) / 2} \\
& \leqslant C_{N} 2^{-j(n-1) / 2}
\end{aligned}
$$

for all $y \in \mathbb{R}^{n}$, since

$$
\gamma \geqslant \frac{1}{\rho} \geqslant \frac{n+\rho}{n \rho+2-\rho}
$$

Therefore,

$$
\int_{\mathbb{R}^{n}}\left|K_{j}(x, y)\right| \mathrm{d} x \leqslant \sum_{\nu} \int_{\mathbb{R}^{n}}\left|K_{j}^{\nu}(x, y)\right| \mathrm{d} x \leqslant \sum_{\nu} C_{N} 2^{-j(n-1) / 2} \leqslant A,
$$

for all $y \in \mathbb{R}^{n}$; this proves (i).

Next we turn our attention to (ii). We begin by noticing that, with computations analogous to the one just made, one can show that for all multi-indices $\alpha$ we have

$$
\int_{\mathbb{R}^{n}}\left|\partial_{y}^{\alpha} K_{j}^{\nu}(x, y)\right| \mathrm{d} x \leqslant C \cdot 2^{|\alpha| j / \gamma} \cdot 2^{-j(n-1) / 2},
$$

for all $y \in \mathbb{R}^{n}$. Now let $q_{j}^{\nu}\left(x, y-y^{\prime}\right)$ be the Taylor polynomial of the function $K_{j}^{\nu}(x, y)$, centred at $y=y^{\prime}$, of order $M-1$. Then

$$
\int_{\mathbb{R}^{n}}\left|K_{j}^{\nu}(x, y)-q_{j}^{\nu}\left(x, y-y^{\prime}\right)\right| \mathrm{d} x \leqslant C \cdot 2^{j M / \gamma} \cdot 2^{-j(n-1) / 2}\left|y-y^{\prime}\right|^{M}
$$

and, summing over $\nu$, we obtain (ii).

Finally, we prove (iii). Let $B$ be the ball of centre $\bar{y}$ and radius $\delta \leqslant 1$, with $j$ such that $2^{-j} \leqslant \delta$. Let $k$ be an integer such that $2^{-k} \leqslant \delta \leqslant 2^{-k+1}$. We claim that there exists a constant $C>0$ such that for every $y \in B$ and $x \in{ }^{c} B^{*}$ we have

$$
\left|2^{j}\left(\phi_{\xi}\left(x, \xi_{j}^{\nu}\right)-y\right)_{1}\right|^{2}+\left|2^{j \rho / 2}\left(\phi_{\xi}\left(x, \xi_{j}^{\nu}\right)-y\right)^{\prime}\right|^{2} \geqslant C 2^{j \rho-k} .
$$

Arguing as before, i.e. performing the same change of variable, we see that

$$
\begin{aligned}
& \int_{{ } B^{*}}\left|K_{j}^{\nu}(x, y)\right| \mathrm{d} x \\
& \quad \leqslant A_{N} \int_{c_{B^{*}}} \frac{2^{j(2-(n-\rho)+(n-1)(2-\gamma)) / 2 \gamma}}{\left(1+4 \pi^{2}\left|2^{j}\left(\phi_{\xi}\left(x, \xi_{j}^{\nu}\right)-y\right)_{1}\right|^{2}+4 \pi^{2}\left|2^{j \rho / 2}\left(\phi_{\xi}\left(x, \xi_{j}^{\nu}\right)-y\right)^{\prime}\right|^{2}\right)^{N}} \mathrm{~d} x \\
& \quad \leqslant A_{N} \int_{\mathbb{R}^{n}} \frac{2^{j(2-(n-\rho)+(n-1)(2-\gamma)) / 2 \gamma} \cdot 2^{-j \rho+k}}{\left(1+4 \pi^{2}\left|2^{j}(z-y)_{1}\right|^{2}+4 \pi^{2}\left|2^{j \rho / 2}(z-y)^{\prime}\right|^{2}\right)^{N-1}} \mathrm{~d} z
\end{aligned}
$$




$$
\begin{aligned}
& \leqslant A_{N} \int_{\mathbb{R}^{n}} \frac{2^{j(2-(n-\rho)+(n-1)(2-\gamma)-2 \gamma-(n-1) \rho \gamma) / 2 \gamma} \cdot 2^{-j \rho+k}}{\left(1+4 \pi^{2}|u|^{2}\right)^{N-1}} \mathrm{~d} u \\
& \leqslant A_{N} 2^{-j(n-1) / 2} \cdot 2^{-j \rho} \delta^{-1}
\end{aligned}
$$

for $y \in B$ and $2^{j} \leqslant \delta$. Finally, summing in $\nu$ we obtain the desired conclusion.

We now complete the proof of Theorem 2.4. We estimate the second integral on the right-hand side of (3.6). We split the integration region into two parts, one where $|T a| \leqslant 1$ and one where $|T a|>1$. In the first case we can estimate the integral with $|\operatorname{supp}(b)|$. Thus, we may assume that $|T a|>1$ in the region of integration, so that

$$
\int_{{ }^{B^{*}}}|T a(x)|^{p} \mathrm{~d} x \leqslant \int_{{ }^{B_{B}}}|T a(x)| \mathrm{d} x .
$$

Set

$$
\beta=\frac{1}{\rho}\left(1+n\left(\frac{1}{p}-1\right)\right)
$$

Then

$$
\int_{{ }^{c} B^{*}}|T a(x)| \mathrm{d} x \leqslant \int_{c^{*} B^{*}}\left|T_{0} a(x)\right| \mathrm{d} x+\sum_{2^{j}<\delta^{-\beta}} \int_{c^{*}}\left|T_{j} a(x)\right| \mathrm{d} x+\sum_{2^{j} \geqslant \delta^{-\beta}} \int_{c^{*} B^{*}}\left|T_{j} a(x)\right| \mathrm{d} x .
$$

We begin with the last term on the right-hand side. Note that $2^{-j} \leqslant \delta^{\beta} \leqslant \delta$. Using Proposition 3.2 (iii) we have

$$
\begin{aligned}
\sum_{2^{j} \geqslant \delta^{-\beta}} \int_{c_{B^{*}}}\left|T_{j} a(x)\right| \mathrm{d} x & \leqslant \sum_{2^{j} \geqslant \delta^{-\beta}} \int_{B} \int_{c_{B^{*}}}\left|K_{j}(x, y)\right| \mathrm{d} x|a(y)| \mathrm{d} y \\
& \leqslant A \sum_{2^{j} \geqslant \delta^{-\beta}} \frac{2^{-j \rho}}{\delta} \int_{B}|a(y)| \mathrm{d} y \\
& \leqslant A \sum_{2^{j} \geqslant \delta^{-\beta}} \frac{2^{-j \rho}}{\delta} \delta^{n(1-1 / p)} \\
& \leqslant A \cdot \delta^{\beta \rho-1+n(1-1 / p)} \\
& \leqslant A
\end{aligned}
$$

because of our choice of $\beta$.

Now we consider the other sum in (3.14). We use the moment condition of the atom $a$, which holds since $\delta \leqslant 1$. Using Proposition 3.2 (ii) we have

$$
\begin{aligned}
\sum_{2^{j}<\delta^{-\beta}} \int_{c^{*} B^{*}}\left|T_{j} a(x)\right| \mathrm{d} x & =\sum_{2^{j}<\delta^{-\beta}} \int_{c^{*} B^{*}}\left|\int_{B}\left(K_{j}(x, y)-q_{j}(x, y-\bar{y})\right) a(y) \mathrm{d} y\right| \mathrm{d} x \\
& \leqslant \sum_{2^{j}<\delta^{-\beta}} \int_{B} \int_{c^{*} B^{*}}\left|K_{j}(x, y)-q_{j}(x, y-\bar{y})\right| \mathrm{d} x|a(y)| \mathrm{d} y
\end{aligned}
$$




$$
\begin{aligned}
& \leqslant \sum_{2^{j}<\delta^{-\beta}} 2^{j M / \gamma} \int_{B}|y-\bar{y}|^{M}|a(y)| \mathrm{d} y \\
& \leqslant \sum_{2^{j}<\delta^{-\beta}} 2^{j M / \gamma} \cdot \delta^{M+n(1-1 / p)} \\
& \leqslant A \cdot \delta^{-\beta M / \gamma} \cdot \delta^{M+n(1-1 / p)} \\
& \leqslant A
\end{aligned}
$$

recalling that $M=[n(1 / p-1)]+1$ and that $\gamma=M \beta(M+n(1-1 / p))^{-1}$.

We finally estimate $\int_{c_{B^{*}}}\left|T_{0} a(x)\right| \mathrm{d} x$. We note that, since $\delta \leqslant 1, a$ is also an atom in $H^{p}\left(\mathbb{R}^{n}\right)$. Therefore, $\hat{a} \in \mathcal{C}\left(\mathbb{R}^{n}\right)$ and $|\hat{a}(\eta)| \leqslant c_{n, p}|\eta|^{n(1 / p-1)}$ (see, for example, $[\mathbf{1 7}$, $\S$ III.5.4]), while the Fourier transform of $b_{0}(x, y, \xi) \exp (-\mathrm{i} y \cdot \xi)$ in $y$ is a Schwartz function in all of its arguments. Then, by Parseval, if $N$ is large enough,

$$
\begin{aligned}
\int_{{ }^{B^{*}}}\left|T_{0} a(x)\right| \mathrm{d} x & =\int_{c_{B^{*}}}\left|\int_{\mathbb{R}^{n}} \int_{|\xi| \leqslant 2^{1 / \gamma}} b_{0}(x, y, \xi) \mathrm{e}^{2 \pi \mathrm{i}(\phi(x, \xi)-y \cdot \xi)} a(y) \mathrm{d} y \mathrm{~d} \xi\right| \mathrm{d} x \\
& \leqslant A_{N} \int_{c_{B^{*}}} \int_{|\xi| \leqslant 2^{1 / \gamma}} \int_{\mathbb{R}^{n}}(1+|\eta|)^{-N}|\hat{a}(\eta)| \mathrm{d} \eta \mathrm{d} \xi \mathrm{d} x \\
& \leqslant A .
\end{aligned}
$$

This completes the estimate for $\int_{{ }^{B^{*}}}|T a(x)|^{p} \mathrm{~d} x$ and the proof of Theorem 2.4.

\section{Proof of Theorem 2.2}

In order to prove Theorem 2.2 we use Theorem 2.4 and the characterization of the local Hardy spaces stated below. The local Riesz transforms $r_{k}$ are defined as follows. Let $\hat{\psi}_{0}$ be as in (3.11). For $k=1, \ldots, n$ set

$$
\widehat{\left(r_{k} f\right)}(\xi)=\hat{f}(\xi)\left(1-\hat{\psi}_{0}(\xi)\right)\left(-\frac{\mathrm{i} \xi_{k}}{|\xi|}\right) .
$$

Moreover, we say that a tempered distribution is bounded if $f * \Psi \in L^{\infty}\left(\mathbb{R}^{n}\right)$ for all $\Psi \in \mathcal{S}\left(\mathbb{R}^{n}\right)$. Then we have the following characterization of the local Hardy spaces (cf. [17, $\S$ III.4.3] for the analogous characterization for the classical Hardy spaces $\left.H^{p}\left(\mathbb{R}^{n}\right)\right)$. We denote by $\breve{\psi}$ the inverse Fourier transform of a tempered distribution $\psi$.

Proposition 4.1. Let $f$ be a tempered distribution which is bounded. Let $\Psi \in$ $\mathcal{C}_{0}^{\infty}\left(\mathbb{R}^{n}\right), \int \Psi \mathrm{d} x=1$ be fixed. Then $f \in h^{p}\left(\mathbb{R}^{n}\right)$ if and only if $\check{\psi}_{0} * f \in h^{p}\left(\mathbb{R}^{n}\right)$ and there exists a constant $A>0$ such that for all $\varepsilon$ with $0<\varepsilon \leqslant 1$ we have

$$
\sum_{|\alpha| \leqslant M}\left\|r^{\alpha}(f) * \Psi_{\varepsilon}\right\|_{L^{p}\left(\mathbb{R}^{n}\right)} \leqslant A
$$

Here $r^{\alpha}$, as usual, denotes the composition $r_{1}^{\alpha_{1}} \circ \cdots \circ r_{n}^{\alpha_{n}}, M=[n(1 / p-1)]+1$, and $\Psi_{\varepsilon}(x)=\varepsilon^{-n} \Psi(x / \varepsilon)$. 
The proof of this proposition is somewhat standard, although not quite immediate. The authors have not found it in the literature and they give details in [13].

Note that the distribution $T_{b} f$ has compact support, so that $\check{\psi} *\left(T_{b} f\right) \in \mathcal{S}\left(\mathbb{R}^{n}\right)$; hence, $\check{\psi} *\left(T_{b} f\right) \in h^{p}$. Thus, given this characterization, it suffices to prove the following result.

Proposition 4.2. Let $P=P_{\tau}$ be the pseudodifferential operator with symbol $\tau \in S_{1,0}^{\mu^{\prime}}$ given by

$$
\left(P_{\tau} f\right)(x)=\int_{\mathbb{R}^{n}} \tau(x, \xi) \exp (2 \pi \mathrm{i} x \cdot \xi) \hat{f}(\xi) \mathrm{d} \xi .
$$

Then, the composition of $P$ with the Fourier integral operator $T_{b}$ can be written as sum

$$
P_{\tau} T_{b}=F+E,
$$

where $F$ is a Fourier integral operator with the same phase $\phi(x, \eta)$ as $T_{b}$ and symbol $p \in S_{\rho, 1-\rho}^{\mu+\mu^{\prime}}$, and $E$ is a smoothing linear operator in the sense that

$$
E: h^{p}\left(\mathbb{R}^{n}\right) \rightarrow L_{s}^{p}\left(\mathbb{R}^{n}\right)
$$

for every $s \geqslant 0$.

Proof of Proposition 4.2. Let $R>0$ be large enough so that $\operatorname{supp}_{x}(b) \subset B(0, R)$. Let $\chi \in \mathcal{C}_{0}^{\infty}\left(\mathbb{R}^{n}\right)$ be identically 1 on $B(0,2 R)$ and with support contained in $B(0,3 R)$. Let

$$
\tau_{(0)}(x, \xi)=\chi(x) \tau(x, \xi) \quad \text { and } \quad \tau_{(\infty)}(x, \xi)=(1-\chi(x)) \tau(x, \xi) .
$$

We initially assume that $\tau$ has compact support in the variable $\xi$. This assumption allows us to perform changes in the integration orders and guarantees that the expressions that we write are well defined. However, the estimates will not depend on the size of the $\xi$-support. Thus, by a limiting argument, we will be able to remove the compactness assumption at the end of the proof.

We write

$$
\begin{aligned}
&\left(P_{\tau} T_{b}\right) f(x)=\iint \tau_{(0)}(x, \xi) \exp (2 \pi \mathrm{i}(x-y) \cdot \xi) T_{b} f(y) \mathrm{d} \xi \mathrm{d} y \\
&+\iint \tau_{(\infty)}(x, \xi) \exp (2 \pi \mathrm{i}(x-y) \cdot \xi) T_{b} f(y) \mathrm{d} \xi \mathrm{d} y \\
&=\iint_{|\eta|>1}\left(\iint \tau_{(0)}(x, \xi) \exp (2 \pi \mathrm{i}[(x-y) \cdot \xi+\phi(y, \eta)]) b(y, z, \eta) \mathrm{d} \xi \mathrm{d} y\right) \\
& \quad \times f(z) \exp (-2 \pi \mathrm{i} z \cdot \eta) \mathrm{d} \eta \mathrm{d} z \\
&+\iint_{|\eta| \leqslant 1}\left(\iint \tau_{(0)}(x, \xi) \exp (2 \pi \mathrm{i}[(x-y) \cdot \xi+\phi(y, \eta)]) b(y, z, \eta) \mathrm{d} \xi \mathrm{d} y\right) \\
& \quad \times f(z) \exp (-2 \pi \mathrm{i} z \cdot \eta) \mathrm{d} \eta \mathrm{d} z
\end{aligned}
$$




$$
\begin{aligned}
& +\iint_{|\eta|>1}\left(\iint \tau_{(\infty)}(x, \xi) \exp (2 \pi \mathrm{i}[(x-y) \cdot \xi+\phi(y, \eta)]) b(y, z, \eta) \mathrm{d} \xi \mathrm{d} y\right) \\
& \quad \times f(z) \exp (-2 \pi \mathrm{i} z \cdot \eta) \mathrm{d} \eta \mathrm{d} z \\
& +\iint_{|\eta| \leqslant 1}\left(\iint \tau_{(\infty)}(x, \xi) \exp (2 \pi \mathrm{i}[(x-y) \cdot \xi+\phi(y, \eta)]) b(y, z, \eta) \mathrm{d} \xi \mathrm{d} y\right) \\
& \quad \times f(z) \exp (-2 \pi \mathrm{i} z \cdot \eta) \mathrm{d} \eta \mathrm{d} z \\
& =: \sum_{j=1}^{4}\left(T^{(j)} f\right)(x) .
\end{aligned}
$$

It is a well-known fact that $T^{(1)}$ is a Fourier integral operator with the same phase $\phi(x, \eta)$ as $T_{a}$ and symbol $p$ given by

$$
p(x, z, \eta)=\exp (-2 \pi \mathrm{i} \phi(x, \eta)) P_{\tau_{(0)}}(b(\cdot, z, \eta) \exp (2 \pi \mathrm{i} \phi(\cdot, \eta)))(x)
$$

for $|\eta|>0$ (see, for example, [18, Theorem 3.1]). We remark that $p$ is (asymptotically equivalent to a symbol) in $S_{\rho, 1-\rho}^{\mu+\mu^{\prime}}$ and that it has compact support in $x$ and $z$. (The fact that $p \in S_{\rho, 1-\rho}^{\mu+\mu^{\prime}}$ can be seen as in the proof of [18, Theorem 3.1].)

Next we turn to $T^{(2)}$. We wish to prove that

$$
\left\|\partial_{x}^{\alpha}\left(T^{(2)} f\right)\right\|_{L^{p}} \leqslant C\|f\|_{h^{p}},
$$

where the constant $C$ depends only on the orders $\mu, \mu^{\prime}, n, p,|\alpha|$ and on the size of the support of $b$.

Using the assumption (that we will remove later) that $\tau$ has compact support in $\xi$ and an integration by parts, for any non-negative integer $N$ we write

$$
\begin{aligned}
\left(T^{(2)} f\right)(x) & =\iint \tau_{(0)}(x, \xi) \exp (2 \pi \mathrm{i}(x-y) \cdot \xi) T_{b_{0}} f(y) \mathrm{d} y \mathrm{~d} \xi \\
& =\int\left(\int L_{y}^{N}\left(T_{b_{0}} f\right)(y) \mathrm{d} y\right) \frac{\tau_{(0)}(x, \xi)}{\left(1+4 \pi^{2}|\xi|^{2}\right)^{N}} \exp (2 \pi \mathrm{i}(x-y) \cdot \xi) \mathrm{d} \xi
\end{aligned}
$$

where

$$
T_{b_{0}} f(y)=\iint_{|\eta| \leqslant 1} b(y, z, \eta) \exp (2 \pi \mathrm{i}(\phi(y, \eta)-z \cdot \eta)) f(z) \mathrm{d} \eta \mathrm{d} z,
$$

and $L_{y}=I-\Delta_{y}$.

Now we claim that there exists a constant $A=A_{N}$ such that, for all $f \in h^{p}\left(\mathbb{R}^{n}\right)$ and $y \in \operatorname{supp}(b)$,

$$
\left|L_{y}^{N}\left(T_{b_{0}} f\right)(y)\right| \leqslant A\|f\|_{h^{p}} .
$$

Assume the claim for now. Then, if we take $N>\left(\mu^{\prime}+n\right) / 2$

$$
\begin{aligned}
\left|\left(T^{(2)} f\right)(x)\right| & \leqslant \int_{\mathbb{R}^{n}}\left(\int_{\mathbb{R}^{n}}\left|L_{y}^{N}\left(T_{b_{0}} f\right)(y)\right| \mathrm{d} y\right) \frac{\left|\tau_{0}(x, \xi)\right|}{\left(1+4 \pi^{2}|\xi|^{2}\right)^{N}} \mathrm{~d} \xi \\
& \leqslant C\|f\|_{h^{p}}
\end{aligned}
$$


where the constant depends on the seminorm of $\tau$ but not on the size of the support. Therefore,

$$
\left\|T^{(2)} f\right\|_{L^{p}} \leqslant A\|f\|_{h^{p}} .
$$

It is now easy to see that we can apply the same argument to $\partial_{x}^{\alpha}\left(T^{(2)} f\right)(x)$ to obtain the desired estimate (4.1).

Before proving the claim, we also analyse the term $T^{(4)}$. We have

$$
\begin{aligned}
\left(T^{(4)} f\right)(x)= & \iint_{|\eta| \leqslant 1}\left(\iint \tau_{(\infty)}(x, \xi) \exp (2 \pi \mathrm{i}[(x-y) \cdot \xi-\phi(y, \eta)]) b(y, z, \eta) \mathrm{d} \xi \mathrm{d} y\right) \\
& \quad \times \exp (-2 \pi \mathrm{i} z \cdot \eta) f(z) \mathrm{d} \eta \mathrm{d} z \\
= & \int_{|y| \leqslant R} K_{\infty}(x, y-x)\left(T_{b_{0}} f\right)(y) \mathrm{d} y,
\end{aligned}
$$

where $T_{b_{0}}$ is defined as above, and $K_{\infty}(x, y-x)$ is the kernel of the pseudodifferential operator with symbol $\tau_{(\infty)}(x, \xi)=(1-\chi(x)) \tau(x, \xi)$. It is well known that $K_{\infty} \in \mathcal{C}^{\infty}\left(\mathbb{R}^{n} \times\right.$ $\left.\mathbb{R}^{n} \backslash\{0\}\right)$ and that there exists a constant $C=C_{\alpha, \beta}>0$ such that

$$
\left|\partial_{x}^{\beta} \partial_{\xi}^{\alpha} K_{\infty}(x, z)\right| \leqslant C|z|^{-n-\mu^{\prime}-|\alpha|-N},
$$

for $z \neq 0$, when $n+\mu^{\prime}+|\alpha|+N>0$.

Note that $K_{\infty}(x, y-x)\left(T_{b_{0}} f\right)(y) \neq 0$ implies $|x-y|>R$. Therefore, using the claim again, we have

$$
\begin{aligned}
\left\|\partial_{x}^{\beta}\left(T^{(4)} f\right)\right\|_{L^{p}}^{p} & =\int_{|x|>2 R}\left|\int_{|y| \leqslant R} \partial_{x}^{\beta} K_{\infty}(x, x-y)\left(T_{b_{0}} f\right)(y) \mathrm{d} y\right|^{p} \mathrm{~d} x \\
& \leqslant C\|f\|_{h^{p}}^{p} \int_{|x|>2 R}\left(\int_{|y| \leqslant R}|x-y|^{-n-\mu^{\prime}-N} \mathrm{~d} y\right)^{p} \mathrm{~d} x \\
& \leqslant C\|f\|_{h^{p}}^{p} \int_{|x|>2 R}|x|^{-\left(n+\mu^{\prime}+N\right) p} \mathrm{~d} x \\
& \leqslant C\|f\|_{h^{p}}^{p}
\end{aligned}
$$

if we choose $N$ large enough.

Finally, we consider $T^{(3)}$. It is easy to see that

$$
\left(T^{(3)} f\right)(x)=\iint_{|\eta|>1} p(x, z, \eta) \exp (2 \pi \mathrm{i}(x-z) \cdot \eta) f(z) \mathrm{d} \eta \mathrm{d} z,
$$

where

$$
p(x, z, \eta)=\exp (-2 \pi \mathrm{i} x \cdot \eta) \int_{|y| \leqslant R,|y-x| \geqslant R} K_{\infty}(x, x-y) b(y, z, \eta) \exp (2 \pi \mathrm{i} \phi(y, \eta)) \mathrm{d} y .
$$

It is a standard argument to check that $p \in S_{\rho, 1-\rho}^{-N}$ for all $N>0$, using the fact that $\phi$ is $\mathcal{C}^{\infty}$ in $\eta$ for $|\eta|>0$. Then, it is a standard fact that it maps $h^{p}\left(\mathbb{R}^{n}\right)$ into $L_{s}^{p}\left(\mathbb{R}^{n}\right)$ for all $s \geqslant 0$. 
It remains only to prove the claim (4.2). It suffices to prove that, for every $p$-atom $a$ in $h^{p}$, with support contained in a ball $B_{\delta}$ of radius $\delta$,

$$
\left|\iint_{|\eta| \leqslant 1} L_{y}^{N} b(y, z, \eta) \exp (2 \pi \mathrm{i}(\phi(y, \eta)-z \cdot \eta)) a(z) \mathrm{d} \eta \mathrm{d} z\right| \leqslant C,
$$

where $C$ is independent of $\delta$.

Let

$$
g(y, \theta)=\left(\int_{|\eta| \leqslant 1} L_{y}^{N} b(y, \cdot, \eta) \exp (2 \pi \mathrm{i}(\phi(y, \eta)-(\cdot) \cdot \eta) \mathrm{d} \eta)^{\hat{n}}(\theta) .\right.
$$

Then $g$ is $C^{\infty}$ and compactly supported in $y$, while it is $C^{\infty}$ and rapidly decreasing in $\theta$.

If $\delta \leqslant 1$, then $a$ is also an atom of $H^{p}\left(\mathbb{R}^{n}\right)$ and $|\hat{a}(\theta)| \leqslant c_{n, p}|\theta|^{n(1 / p-1)}$ (see, for example, $[17, \S$ III.5.4]). Then

$$
\begin{aligned}
\mid \iint_{|\eta| \leqslant 1} L_{x}^{N}(b(y, z, \eta) \exp (2 \pi \mathrm{i}(\phi(x, \eta)-z & \cdot \eta))) a(z) \mathrm{d} \eta \mathrm{d} z \mid \\
& \leqslant C_{N} \int(1+|\theta|)^{-N}|\theta|^{n(1 / p-1)} \mathrm{d} \theta \\
& \leqslant C
\end{aligned}
$$

uniformly in $\delta$.

If $\delta>1$, then

$$
\begin{aligned}
\left|\iint_{|\eta| \leqslant 1} L_{x}^{N}(b(y, z, \eta) \exp (2 \pi \mathrm{i}(\phi(x, \eta)-z \cdot \eta))) a(z) \mathrm{d} \eta \mathrm{d} z\right| & \leqslant C\|a\|_{L^{1}} \\
& \leqslant C \delta^{n(1-1 / p)} \\
& \leqslant C .
\end{aligned}
$$

This proves the claim and hence the proposition.

We are now ready to finish the proof of Theorem 2.2. In view of Proposition 4.1, it suffices to show that

$$
\sum_{|\alpha| \leqslant M}\left\|r^{\alpha}\left(T_{b} f\right) * \Psi_{\varepsilon}\right\|_{L^{p}\left(\mathbb{R}^{n}\right)} \leqslant A
$$

for every $f \in h^{p}\left(\mathbb{R}^{n}\right)$, with $A$ independent of $\varepsilon$.

We note that we can write

$$
r^{\alpha}\left(T_{b} f\right) * \Psi_{\varepsilon}=P_{\varepsilon} T_{b},
$$

where $P_{\varepsilon}$ is a pseudodifferential operator of order 0 (and type $(1,0)$ ). Thus, by Proposition 4.2 ,

$$
P_{\varepsilon} T_{b}=F_{\varepsilon}+E_{\varepsilon}
$$

A careful analysis of the symbols of the operators $F_{\varepsilon}+E_{\varepsilon}$ reveals that these operators are bounded on $h^{p}\left(\mathbb{R}^{n}\right)$ with norms uniformly bounded in $\varepsilon$. Hence, for $f \in h^{p}\left(\mathbb{R}^{n}\right)$ and 
for every $|\alpha| \leqslant M$,

$$
\begin{aligned}
\left\|r^{\alpha}\left(T_{b} f\right) * \Psi_{\varepsilon}\right\|_{L^{p}} & =\left\|P_{\varepsilon} T_{b}(f)\right\|_{L^{p}} \\
& \leqslant\left\|F_{\varepsilon}(f)\right\|_{L^{p}}+\left\|E_{\varepsilon}(f)\right\|_{L^{p}} \\
& \leqslant A\|f\|_{h^{p}}
\end{aligned}
$$

with the constant $A$ independent of $\varepsilon$. This proves the theorem.

\section{The case of manifolds of bounded geometry}

In this final section we extend our main result to the case of a smooth, complete Riemannian manifold with bounded geometry. Since our main result, Theorem 2.1, is already local in nature, it suffices to choose smooth manifolds on which the local Hardy spaces are well defined and admit an atomic decomposition.

A smooth, complete, connected Riemannian manifold $X$ is said to be of bounded geometry if its radius of injectivity $r_{\text {in }}$ is positive and if every covariant derivative of the curvature tensor is bounded. Examples of manifolds of bounded geometry are the compact manifolds and homogeneous manifolds, e.g. Lie groups (see [9]).

Theorem 5.1. Let $X$ and $Y$ be smooth, connected, complete Riemannian manifolds with bounded geometry of the same dimension $n$. Let $\mathcal{C} \subseteq T^{*}(X) \backslash\{0\} \times T^{*}(Y) \backslash\{0\}$ be a submanifold which is locally a canonical graph and let $\mathcal{F} \in I^{\mu}(X \times Y, \mathcal{C})$. Let $h^{p}$ denote the local Hardy space for $0<p \leqslant 1$ and let $\mu \leqslant-(n-1)(1 / p-1 / 2)$. Then

$$
\tilde{\mathcal{F}}: h_{\text {comp }}^{p}(X) \rightarrow h_{\mathrm{loc}}^{p}(Y)
$$

is bounded.

On such manifolds, it is possible to define the local Hardy spaces and show that they admit an atomic decomposition analogous to that in the Euclidean space $\mathbb{R}^{n}$ (see [15]). Once the atomic decomposition for the local Hardy spaces is available, our main result, Theorem 2.1, immediately extends to this setting.

We remark that in [16] Skrzypczak studied the boundedness of classical pseudodifferential operators on various scales of spaces, including the local Hardy spaces.

Acknowledgements. This work was done within the project COFIN 2001 'Analisi Armonica' and the IHP project 'HARP'. We thank the European Commission for the support provided.

\section{References}

1. R. Beals, Spatially inhomogeneous pseudodifferential operators, II, Commun. Pure Appl. Math. 27 (1974), 161-205.

2. J. J. Duistermat, Fourier integral operators, Progress in Mathematics, Volume 130 (Birkhäuser, Boston, MA, 1996).

3. G. I. Eskin, Degenerate elliptic pseudo-differential operators of principal type. Mat. Sb. 82 (1970), 585-628 (in Russian) (English transl.: Math. Sb. 11 (1970), 539-582). 
4. D. Goldberg, A local version of real Hardy spaces, Duke Math. J. 46 (1979), 27-42.

5. A. Greenleaf and G. Uhlmann, Estimates for singular Radon transforms and pseudodifferential operators with singular symbols, J. Funct. Analysis 89 (1990), 202-232.

6. A. GRIGis AND J. SJÖstrand, Microlocal analysis for differential operators: an introduction, London Mathematical Society Lecture Note Series, Volume 196 (Cambridge University Press, 1994).

7. L. Hörmander, Fourier integral operators, I, Acta Math. 127 (1971), 79-183.

8. L. HÖRMANDER, The analysis of linear partial differential operators, IV, Grundlehren der Mathematichen Wissenschaften, Volume 275 (Springer, 1994).

9. J. Jost, Riemannian geometry and geometric analysis, 4th edn (Springer, 2005).

10. S. G. Krantz, Fractional integration on Hardy spaces, Studia Math. 73 (1982), 87-94.

11. W. Littman, $L^{p}-L^{q}$-estimates for singular integral operators, Proc. Symp. Pure Appl. Math. AMS 23 (1973), 479-481.

12. A. MiYACHI, On some estimates for the wave equation in $L^{p}$ and $H^{p}$, J. Fac. Sci. Univ. Tokyo (1) 27 (1980), 331-354.

13. M. M. Peloso And S. SeCCO, On higher moment characterizations of local Hardy spaces, Collect. Math., in the press.

14. A. Seeger, C. D. Sogge And E. M. Stein, Regularity properties of Fourier integral operators, Annals Math. 134 (1991), 231-251.

15. L. SkrzyPCZAK, Atomic decompositions on manifolds with bounded geometry, Forum Math. 10 (1998), 19-38.

16. L. SKRZYPCZAK, Mapping properties of pseudodifferential operators on manifolds with bounded geometry, J. Lond. Math. Soc. 57 (1998), 721-738.

17. E. M. STEIN, Harmonic analysis, real-varaible methods, orthogonality and oscillatory integrals (Princeton University Press, Princeton, NJ, 1993).

18. F. TREves, Introduction to pseudodifferential and Fourier integral operators, Volume II (Plenum, New York, 1982). 